\title{
Mesenchymal Stem Cells Ameliorate Mitochondrial Dysfunction in a - cells and Hyperglucagonemia in type 2 Diabetes via SIRT1/FoxO3a Signaling
}

Jia Song

Shandong University Qilu Hospital

Lingshu Wang

Shandong University Qilu Hospital

Xinghong Guo

Shandong University Qilu Hospital

Qin He

Shandong University Qilu Hospital

Chen Cui

Shandong University Qilu Hospital

Huiqing $\mathrm{Hu}$

Shandong University Qilu Hospital

Nan Zang

Shandong University Qilu Hospital

Mengmeng Yang

Shandong University Qilu Hospital

Fei Yan

Shandong University Qilu Hospital

Kai Liang

Shandong University Qilu Hospital

Chuan Wang

Shandong University Qilu Hospital

Fuqiang Liu

Shandong University Qilu Hospital

Yujing Sun

Shandong University Qilu Hospital

\section{Falian He}

Nuolai Biomedical Technology Co.Ltd.

\section{Zheng Sun}

Shandong University Qilu Hospital

Xinguo Hou 
Shandong University Qilu Hospital

\section{Hong Lai}

Shandong University Qilu Hospital

\section{Li Chen ( $\nabla$ chenli3@medmail.com.cn )}

Shandong University Qilu Hospital https://orcid.org/0000-0001-7670-8062

\section{Research}

Keywords: a-cells, hucMSCs, glucagon secretion, mitochondrial function, SIRT1/FoxO3a

Posted Date: October 29th, 2021

DOI: https://doi.org/10.21203/rs.3.rs-1002080/v1

License: (c) (1) This work is licensed under a Creative Commons Attribution 4.0 International License. Read Full License 


\section{Abstract}

Background: Dysregulation of a-cells results in hyperglycemia and hyperglucagonemia in type 2 diabetes mellitus (T2DM). Mesenchymal stem cell (MSC)-based therapy increases oxygen consumption of islets and enhances insulin secretion. However, the underlying mechanism for the protective role of MSCs in acell mitochondrial dysfunction remains unclear. Here, we evaluated the efficacy and molecular mechanisms of human umbilical cord MSCs (hucMSCs) on a-cell mitochondrial function and glucagon secretion in T2DM.

Methods: hucMSCs were used to treat two kinds of T2DM mice and aTC1-6 cells to explore the role of hucMSCs in improving a-cell mitochondrial dysfunction and hyperglucagonemia. Plasma and supernatant glucagon were detected by enzyme-linked immunosorbent assay (ELISA). Mitochondrial function of a-cells was assessed by the Seahorse Analyzer. To investigate the underlying mechanisms, Sirtuin 1 (SIRT1), Forkhead box 03a (FoxO3a), glucose transporter type1 (GLUT1), and glucokinase (GCK) were assessed by western blotting analysis.

Results: In vivo, hucMSC infusion improved glucose and insulin tolerance, as well as hyperglycemia and hyperglucagonemia in T2DM mice. Meanwhile, hucMSC intervention rescued islet structure and decreased $a$ - to $\beta$-cell ratio. Consistently, glucagon secretion from aTC1- 6 cells was inhibited by hucMSCs in vitro. Meanwhile, hucMSC treatment activated intracellular SIRT1/FoxO3a signaling, promoted glucose uptake and activation, alleviated mitochondrial dysfunction, and enhanced ATP production. However, transfection of SIRT1 small interfering RNA (siRNA) or the application of SIRT1 inhibitor EX-527 weakened the therapeutic effects of hucMSCs on mitochondrial function and glucagon secretion.

Conclusions: Our observations indicate that hucMSCs mitigate mitochondrial dysfunction and glucagon hypersecretion of a-cells in T2DM via SIRT1/FoxO3a signaling, which provides novel evidence demonstrating the potential for hucMSCs in treating T2DM.

\section{Introduction}

Pancreatic a-cells release glucagon and promote glucose output from the liver [1]. Relative hyperglucagonemia caused by a-cell dysregulation contributes to fasting and postprandial hyperglycemia in patients with type 2 diabetes mellitus (T2DM) [2]. Single-cell transcriptomes from cultured pancreatic islets of T2DM donors have revealed that genes responsible for energy metabolism in mitochondria are significantly downregulated in a-cells [3]. Defective mitochondrial metabolism in a-cells accounts for dysregulated glucagon secretion in T2DM [4].

Sirtuins regulate metabolism by controlling mitochondrial function, which is closely related to T2DM, particularly Sirtuin 1 (SIRT1) [5]. SIRT1, a nicotinamide adenine dinucleotide (NAD+)-dependent histone deacetylase, participates in mitochondrial biogenesis and oxidative metabolism [6, 7]. In pancreatic $\beta$ cells, SIRT1 enhances glucose-stimulated insulin secretion [8-10]. The deletion of SIRT1 in mouse $\beta$-cells 
impairs insulin secretion by disrupting glucose sensing [11]. However, studies showing the role of SIRT1 in pancreatic a-cell mitochondrial function are limited.

Mesenchymal stem cell (MSC)-based therapy is one of the most promising and effective approaches for treating diabetes [12]. Human umbilical cord MSCs (hucMSCs) can be isolated at ease with few ethical issues and have high proliferative potential and lower immunogenicity compared with other MSCs, and this makes hucMSCs an ideal choice in diabetes treatment $[13,14]$. Studies have shown that MSC intervention is effective in many diseases by regulating mitochondrial function such as myocardial infarction and diabetic endothelial dysfunction [15-17]. Interestingly, MSCs increase oxygen consumption in islets and enhance insulin secretion [18]. Our previous study demonstrated that MSCconditioned medium alleviates high-fat diet (HFD)-induced hyperglucagonemia [19]. However, whether MSCs regulate a-cell mitochondrial function in T2DM remains unclear.

Therefore, in this study, we used two kinds of T2DM mice and aTC1-6 cells pre-exposed to palmitate (PA) to explore the effects and underlying mechanism of hucMSCs in improving a-cell mitochondrial dysfunction and hyperglucagonemia.

\section{Materials And Methods}

\section{Cell culture and treatments}

With the approval of the Ethics Committee of Shandong University Qilu Hospital, we obtained fresh human umbilical cords from healthy newborns. All of the participants gave informed consent for the use of umbilical cords in this study. After washing the umbilical cords for thrice in normal saline, we removed blood vessels and cut Wharton's jelly into small pieces with sterile scissors. These pieces were then spread in cell culture flasks with a-MEM medium (Gibco, USA) containing $10 \%$ fetal bovine serum (FBS; Gibco) and $100 \mathrm{U} / \mathrm{mL}$ penicillin and $100 \mu \mathrm{g} / \mathrm{mL}$ streptomycin (Gibco). Cultures were maintained at $37^{\circ} \mathrm{C}$ in a humidified atmosphere of $5 \% \mathrm{CO}_{2}$ in air. We changed the culture medium every $3 \mathrm{~d}$ until the cells reached $80 \%$ confluency. The third to the fifth passage of cells were used for identification, administration, and Transwell ${ }^{\mathrm{TM}}$ co-culturing.

Human embryo lung fibroblasts (HELFs) were obtained from the China Cell Culture Center (Shanghai, China) and cultured in Dulbecco's modified Eagle's medium (DMEM)/high-glucose medium (Gibco, USA) supplemented with $10 \% \mathrm{FBS}, 100 \mathrm{U} / \mathrm{mL}$ penicillin and $100 \mu \mathrm{g} / \mathrm{mL}$ streptomycin at $37^{\circ} \mathrm{C}$ and in a $5 \% \mathrm{CO}_{2}$ incubator.

aTC1-6 cells were obtained from the American Type Culture Collection (ATCC Number: CRL-2934 ${ }^{\mathrm{TM}}$ ) and cultured in DMEM/high-glucose medium (Gibco, USA) supplemented with $10 \%$ FBS and antibiotics. After reaching $70-80 \%$ confluency, a-cells were stimulated with PA $(0.5 \mathrm{mM})$ for $48 \mathrm{~h}$, followed by treatment with hucMSCs or HELF for $24 \mathrm{~h}$ via a Transwell ${ }^{\mathrm{TM}}$ system (Catalog No. 3450; Corning, USA). To further 
explore the mechanism of hucMSCs, a-cells were pre-treated with SIRT1 inhibitor (EX-527; $20 \mu \mathrm{M}$, Selleck, Shanghai, China), siR-SIRT1, and siR-FoxO3 before treatment with hucMSCs.

\section{Animal models and hucMSC administration}

Six-week-old male C57BL/6J mice were purchased from the Model Animal Research Center of Shandong University (Jinan, China). The mice were housed with a 12-h light/dark cycle at a temperature $\left(22^{\circ} \mathrm{C}-\right.$ $\left.25^{\circ} \mathrm{C}\right)$ - and humidity ( $55 \pm 5 \%$ )-controlled environment. The T2DM mice model was established by a continuously HFD for 16 weeks followed by a single dose of streptozocin (STZ, $100 \mathrm{mg} / \mathrm{kg}$, Cat. No. S0130; Sigma-Aldrich) intraperitoneally after fasting for $12 \mathrm{~h}$. Four-week-old male $\mathrm{db} / \mathrm{db}$ mice were purchased from Synergy Pharmaceutical Bioengineering Co., Ltd. (Nanjing, China) and were fed a normal chow diet. T2DM was identified as fasting glucose $\geq 16.7 \mathrm{mmol} / \mathrm{L}$ twice in succession. Then, hucMSCs ( $1 \times 10^{\wedge} 6$ cells/mouse) were suspended in PBS and injected into both kinds of T2DM mice via the tail vein every $7 \mathrm{~d}$ for 6 cycles. To evaluate the mechanism of hucMSCs in vivo, HFD and STZ-induced T2DM mice were also administered intraperitoneally with EX-527 $(10 \mathrm{mg} / \mathrm{kg})$ every $3 \mathrm{~d}$ for 6 weeks. All of the animal experiments were approved by the Animal Ethics Committee of Shandong University (Ethical No. DWLL2019-016).

\section{Animal procedures}

The intraperitoneal glucose tolerance test (IPGTT) and intraperitoneal insulin tolerance test (IPITT) were performed after hucMSC administration. Each group contained four to six mice selected via randomization procedure. During IPGTT, tail vein blood glucose levels were measured by Accu-Chek ${ }^{\circledR}$ Performa (Roche Life Science, USA) at 0, 15, 30, 60, 90, 120, and 180 min after i.p. injection of $2 \mathrm{~g} / \mathrm{kg}$ body weight of glucose. During IPITT, tail vein blood glucose levels were measured at 0, 30, 60, 90, 120, and 180 min after i.p. injection of $0.75 \mathrm{U} / \mathrm{kg}$ body weight of insulin. The area under the curve (AUC) was calculated using the trapezoidal rule. After the mice were anaesthetized, their pancreas were fixed in $4 \%$ paraformaldehyde for immunofluorescence staining. The sera of mice were stored at $-80^{\circ} \mathrm{C}$ for glucagon enzyme-linked immunosorbent assay (ELISA; Cloud-Clone, Wuhan, China) and insulin ELISA (ALPCO, USA) test.

\section{Immunofluorescence staining of the pancreas}

The pancreatic tissues that were fixed in $4 \%$ paraformaldehyde were embedded in paraffin, sectioned at $5-\mu \mathrm{m}$ thickness, and mounted on glass slides. The slides were dewaxed, after which antigen retrieval was performed using antigen unmasking buffer. After blocking for $30 \mathrm{~min}$ at room temperature in a proteinblocking solution (10\% normal goat serum), the slides were incubated with anti-mouse insulin antibody (Proteintech, China, Cat. No. 66198-1-Ig, 1:1,000) and anti-rabbit glucagon antibody (Proteintech, Cat. No.15954-1-AP, 1:200) overnight at $4^{\circ} \mathrm{C}$. The slides were then incubated with goat anti-rabbit FITC (Zhongshan, Beijing, China, Cat. No. ZF-0311, 1:200) and goat anti-mouse TRITC (Zhongshan, Cat. No. ZF-0313, 1:200) for $60 \mathrm{~min}$ at room temperature and then stained for DAPI for $5 \mathrm{~min}$. Fluorescence was observed and captured using a fluorescence microscope (Olympus BX53, Japan). The areas of a-cells 
and $\beta$-cells were analyzed by Image-Pro Plus software. The $\alpha$ - or $\beta$-cell ratio in islets was measured by the glucagon- or insulin-positive area divided by the total islet area.

\section{Glucagon secretion}

After different kinds of intervention, supernatants were removed and aTC1-6 cells were balanced in KrebsRinger bicarbonate HEPES (KRBH) buffer ( $120 \mathrm{mM} \mathrm{NaCl}, 0.75 \mathrm{mM} \mathrm{CaCl} \cdot 2 \mathrm{H}_{2} \mathrm{O}, 4 \mathrm{mM} \mathrm{KH}_{2} \mathrm{PO}_{4}, 10 \mathrm{mM}$ $\mathrm{NaHCO}_{3}, 1 \mathrm{mM} \mathrm{MgSO}{ }_{4} \cdot 7 \mathrm{H}_{2} \mathrm{O}, 30 \mathrm{mM} \mathrm{HEPES}, 1 \% \mathrm{BSA}$ ) containing no glucose for $0.5 \mathrm{~h}$ in $37^{\circ} \mathrm{C}$, followed by incubation in KRBH buffer containing $25 \mathrm{mmol} / \mathrm{L}$ glucose for $2 \mathrm{~h}$. Subsequently, supernatants were collected and kept at $-80^{\circ} \mathrm{C}$ for glucagon ELISA (Cloud-Clone, Wuhan, China).

\section{Real-time quantitative PCR analysis}

Total RNA from aTC1-6 cells was extracted using an E.Z.N.A. MicroElute Total RNA Kit (Cat. no. R6831-01; Omega BioTek, USA) following the manufacturer's instructions. Next, 1 mg RNA was reverse-transcribed into cDNA using a Prime Script RT Reagent Kit (Cat. no. RR047A; Takara, Japan). Primers were chemically synthesized by GenePharma (Shanghai, China) Co., Ltd. The primer sequences of proglucagon were sense 5'-GCACATTCACCAGCGACTAC-3' and antisense 5'-CTGGTGGCAAGATTGTCCAG-3'. The primer sequences of $\beta$-actin were sense 5'-AAGAGCTATGAGCTGCCTGA-3' and antisense $5^{\prime}$ -

TACGGATGTCAACGTCACAC-3'. Real-time PCR was conducted with the SYBR Green PCR kit (Cat. No. RR420A; Takara), gene expression changes were determined with the comparative CT $\left(2^{-\Delta \Delta C t}\right)$ method, and quantification was achieved by normalization using $\beta$-actin as control.

\section{Preparation of cell lysate and western blot analysis}

After being washed by ice-cold PBS, aTC1-6 cells were lysed in radioimmunoprecipitation assay (RIPA) lysis buffer (P0013B, Beyotime, Shanghai, China) for approximately $30 \mathrm{~min}$. Then, the samples were centrifuged at $13,500 \mathrm{rpm}$ at $4^{\circ} \mathrm{C}$ for $20 \mathrm{~min}$. Protein concentration was determined using the bicinchoninic acid (BCA) method (Beyotime, China). Subsequently, proteins were separated by $10 \%$ sodium dodecyl sulfate-polyacrylamide gel electrophoresis (SDS-PAGE) (EpiZyme, China) and transferred onto polyvinylidene difluoride (PVDF) membranes (IPVH00010 $0.45 \mu \mathrm{m}$, Millipore, USA). Then, the membranes were blocked with 5\% skim milk in Tris-buffered saline solution containing Tween-20 (SigmaAldrich, USA) for $1 \mathrm{~h}$ at room temperature and incubated in specific primary antibodies at $4^{\circ} \mathrm{C}$ overnight. After detection by horseradish peroxidase-conjugated secondary antibodies for $1 \mathrm{~h}$ at room temperature, the proteins were visualized using enhanced chemiluminescence.

The primary antibodies were as follows: Glucagon(Abcam, Cat. No. ab92517, 1:1,000), SIRT1(Proteintech, China, Cat. No.13161-1-AP, 1:1,000), Fox03a(CST, USA, Cat. No. 2497S, 1:1,000), Glucose transporter type 1(GLUT1, Bioss, China, Cat. No.bs0472R, 1:1,000), Glucokinase(GCK, Abcam, Cat. No. ab88056, 1:1,000), NDUFB8(Proteintech, Cat. No.14794-1-AP, 1:1,000), SDHB(Proteintech, Cat. No.10620-1-AP, 1:5,000), UQCRC2(Proteintech, Cat. No.14742-1-AP, 1:1,000), MTCO2(Proteintech, Cat. No.55070-1-AP, 1:1,000), ATP5A1(Proteintech, Cat. No.14676-1-AP, 1:2,000), HSP90(Santa Cruz, USA, Cat. No.sc13119, 1:500), $\beta$ actin (CST, Cat. No. 3700S, 1:1,000). 


\section{Transmission electron microscopy (TEM)}

aTC1-6 cells were exposed to PA, followed by treatment with hucMSCs or HELFs, and then collected by trypsinization. Afterward, cells were fixed with $2.5 \%$ glutaraldehyde and post-fixed in $1 \%$ phosphatebuffered osmium tetroxide. After being embedded, sectioned, and double-stained with uranyl acetate and lead citrate, electron photomicrographs of aTC1-6 cell ultrastructure were taken via TEM (JEM-1200EX II, JEOL; Tokyo, Japan).

\section{Glucose uptake assay}

Glucose uptake ability of aTC1-6 cells was evaluated using fluorescent glucose 2-NBDG (Maokang Bio, Shanghai, China, Cat. No. MX4511) following the manufacturer's instructions. After intervention, aTC1-6 cells were gently washed with $\mathrm{KRBH}$ and incubated with $50 \mu \mathrm{M} 2-\mathrm{NBDG}$ at $37^{\circ} \mathrm{C}$ for $30 \mathrm{~min}$. Fluorescent intensity of the cells was detected on a microplate reader (Excitation wavelength: $465 \mathrm{~nm}$; Emission wavelength: $540 \mathrm{~nm}$ ).

\section{Determination of intracellular ATP}

Intracellular ATP content was determined using an ATP content kit (Beyotime, China) following the manufacture's instruction. ATP levels were further normalized by protein content.

\section{Seahorse analysis}

The Mito Stress Test Kit (Agilent, Cat. No. 103015-100) was used to detect the oxygen consumption rate (OCR) according to the procedure provided by the manufacturer. aTC1-6 cells were seeded onto each well of an XF96 cell culture microplate at $2{ }^{\star} 10^{\wedge} 4$ cells/well. The OCR was assessed in Seahorse XF DMEM Medium ( $\mathrm{pH}$ 7.4) containing 10-mM glucose, 2-mM pyruvate glutamine, and 1-mM pyruvate according to the manufacturer's instructions. The concentrations of oligomycin, carbonyl cyanide-4-(trifluoromethoxy) phenylhydrazone (FCCP), and antimycin A/rotenone were $1.5,1$, and $0.5 \mu \mathrm{M}$, respectively. OCR was determined and analyzed on the Agilent Seahorse Bioscience XF96 Extracellular Flux Analyzer (Agilent Technologies).

\section{Small interfering RNA transfections}

Small interfering RNA (siRNA) oligonucleotides were synthesized by Shanghai GenePharma Co., Ltd. The sequences of negative control (NC) siRNA were as follows: sense 5'-UUCUCCGAACGUGUCACGUTT-3' and antisense 5'-ACGUGACACGUUCGGAGAATT-3'. The sequences for the SIRT1 siRNA were sense 5'GGGAUCAAGAGGUUGUUAATT-3' and antisense 5'-UUAACAACCUCUUGAUCCCTT-3'. The sequences for the FoxO3 siRNA were as follows: sense 5'-GGAGCUUGGAAUGUGACAUTT-3' and antisense 5'AUGUCACAUUCCAAGCUCCTT-3'.

aTC1-6 cells were transfected with siRNA for SIRT1 and FoxO3 using Lipofectamine 2000 transfection reagent (Invitrogen, USA) according to the manufacturer's instructions. Briefly, the aTC1-6 cells were plated in 6-well plates until they reached $80-90 \%$ confluency. The medium was then removed and 
replaced by Opti-MEM I reduced serum medium (Gibco) mixed with siRNA for SIRT1 and Fox03 (125 nM) for $6 \mathrm{~h}$. Subsequently, the culture medium was replaced by DMEM medium with 10\% FBS, and the cells were treated with hucMSCs. After $24 \mathrm{~h}$, the cells and supernatant were collected for the next experiments.

\section{Statistical analysis}

All of the data were presented as the mean \pm SEM. Differences between two groups were evaluated using unpaired Student's $t$-test; for three groups or more, a one-way ANOVA was performed followed by Bonferroni's test for multiple comparisons. $P<0.05$ was considered to be statistically significant. GraphPad Prism 8 was used to conduct all of the statistical analyses and to create the graphs.

\section{Results}

\section{hucMSC infusion ameliorates hyperglycemia and hyperglucagonemia in HFD and STZ-induced T2DM mice}

Our previous study showed that bone marrow mesenchymal stem cell-conditioned medium (bmMSC-CM) infusion ameliorated HFD-induced hyperglycemia and hyperglucagonemia in mice[19]. To investigate whether hucMSCs exert similar therapeutic effects in T2DM mice, primary hucMSCs were first isolated and identified by their specific surface markers and adipogenic and osteogenic differentiation.

Fluorescence-activated cell sorter (FACS) analysis showed that hucMSCs were positive for stem cell markers CD105 and CD73, and negative for CD34 and HLA-DR (Fig. S1a). Oil Red O staining and Alizarin Red S staining suggested that hucMSCs could differentiate into adipocytes (Fig. S1b) and osteoblasts (Fig. S1c).

Subsequently, to evaluate the effects of hucMSCs on pancreatic a-cells in vivo, we established a T2DM mouse model by HFD and STZ injections and administered hucMSCs into mice via the tail vein. The results of IPGTT and IPITT showed that hucMSC infusion significantly reduced fasting glucose levels and improved glucose tolerance and insulin tolerance. The AUC supported the above findings (Fig. 1a, b). Circulatory glucagon levels markedly increased in the T2DM mice and decreased after hucMSC intervention (Fig. 1C), whereas insulin levels were elevated in hucMSCs group compared to T2DM group (Fig. 1d). Meanwhile, in T2DM mice, the normal structure of islets was destroyed (Fig. 1e) and there was a higher a-cell ratio (Fig. 1f) and a lower $\beta$-cell ratio (Fig. 1g). hucMSC administration partially rescued islet structure and reversed $\alpha$ - and $\beta$-cell imbalance.

\section{hucMSC infusion alleviates hyperglycemia and hyperglucagonemia in $\mathrm{db} / \mathrm{db}$ mice}

To further verify the effects of hucMSCs in T2DM mice, we also injected hucMSCs into $\mathrm{db} / \mathrm{db}$ mice. Similarly, we found that hucMSC infusion reduced fasting glucose levels and improved the glucose tolerance and insulin tolerance during IPGTT and IPITT. The AUC also supported the above findings (Fig. $2 \mathrm{a}, \mathrm{b})$. Circulatory glucagon and insulin levels of $\mathrm{db} / \mathrm{db}$ mice were higher than $\mathrm{db} / \mathrm{m}$ mice, whereas 
hucMSCs intervention ameliorated hyperglucagonemia and hyperinsulinemia (Fig. 2c, d). Moreover, hucMSCs infusion also improved islet structure (Fig. 2e) and reduced a-cell ratio (Fig. 2f) while elevated $\beta$-cell ratio (Fig. 2g). These results suggest that hucMSCs alleviate hyperglycemia and hyperglucagonemia in T2DM mice.

\section{hucMSCs decrease glucagon secretion in aTC1-6 cells}

To observe the effect of hucMSCs on a-cells in vitro, we co-cultured aTC1-6 cells with hucMSCs or HELF via a Transwell ${ }^{\mathrm{TM}}$ system after PA stimulation. ELISA, RT-qPCR, and western blotting were performed for the detection of glucagon levels in supernatants and intracellular glucagon mRNA and protein content. We found that hucMSC treatment remarkably reduced glucagon secretion (Fig. 3a) while promoting intracellular glucagon protein expression (Fig. 3c). There were no significant changes in proglucagon mRNA levels (Fig. 3b). In addition, TEM images showed that there were fewer glucagon granules in the PA group and more granules in the hucMSC group (Fig. 3d), which coincided with intracellular glucagon protein levels. These results demonstrate that hucMSCs regulate glucagon secretion process.

\section{hucMSCs regulate SIRT1/FoxO3a signaling and improve mitochondrial function in aTC1-6 cells}

Upon uptake via GLUTs, glucose is phosphorylated by GCK and participates in mitochondrial oxidation, which results in the accumulation of intracellular ATP. Increased ATP content and the closure of ATPsensitive K-channels ( $\mathrm{K}_{\text {ATP-Channels) }}$ contribute to the suppression of glucagon secretion, which indicates that mitochondrial function plays an important role in a-cells [20-24]. Studies have shown that SIRT1 plays an important role in regulating mitochondrial function and metabolism [6, 7]. Therefore, we explored changes in SIRT1 signaling and mitochondrial function in aTC1- 6 cells. The results showed that PA decreased SIRT1 and its downstream FoxO3a expression (Fig. 4a) and was accompanied by a downregulation of GLUT1 and GCK (Fig. 4b). hucMSC treatment promoted SIRT1 and FoxO3a expression and upregulated the expression of GLUT1 and GCK. Meanwhile, we used fluorescent glucose 2-NBDG to further confirm that hucMSCs increase glucose uptake by a-cells (Fig. 4c). Subsequently, to study the effects of hucMSCs on mitochondrial function, we first detected protein levels of the mitochondrial complex and ATP production. hucMSCs elevated the expression of NDUFB8, SDHB, and MTCO2 (Fig. 4d), which was accompanied by higher intracellular ATP content (Fig. 4e). The results from OCR directly proved that hucMSCs enhance mitochondrial oxidative phosphorylation (OXPHOS), as evidenced by increased maximal respiration (Fig. 4f). These results indicate that hucMSCs promote SIRT1 signaling and improve mitochondrial function in a-cells.

\section{hucMSCs mitigate mitochondrial dysfunction and glucagon hypersecretion by activating SIRT1}

To assess whether hucMSCs regulate mitochondrial function and glucagon secretion through SIRT1, SIRT1 siRNA and the specific inhibitor EX-527 were used to pretreat aTC1-6 cells. In SIRT1 knockdown cells, hucMSC treatment weakly increased SIRT1 and Fox03a expression (Fig. 5a). Moreover, the effects 
of hucMSCs on the upregulation of GLUT1, GCK, and glucose uptake were partially blocked (Fig. 5b, c). In addition, hucMSC therapy did not sufficiently restore mitochondrial OXPHOS and ATP production (Fig. $5 d-f$ ) when SIRT1 was knocked down. Finally, glucagon secretion was not effectively inhibited by hucMSCs (Fig. 5g-i).

Consistently, upregulations of SIRT1 and FoxO3a were partially abolished in aTC1-6 cells after hucMSC therapy when simultaneously treated with SIRT1 inhibitor EX-527 (Fig. 5j). Meanwhile, hucMSCs weakly elevated GLUT1 and GCK expression and glucose uptake when co-treated with EX-527 (Fig. 5k, I). Furthermore, improvement of mitochondrial OXPHOS and ATP production was also weaker in hucMSCs and EX-527 co-treated group compared with the hucMSC-treated group (Fig. 5m-0), which resulted in the restoration of glucagon secretion (Fig. 5p-r). These results demonstrate that hucMSCs mitigate mitochondrial dysfunction and glucagon hypersecretion by activating SIRT1.

\section{Fox03a is involved in the improvement of mitochondrial dysfunction and glucagon hypersecretion by hucMSCs}

To verify whether SIRT1 downstream factor FoxO3a is involved in the regulation of mitochondrial function and glucagon secretion by hucMSCs, FoxO3 siRNA was transfected into aTC1-6 cells. When FoxO3 was knocked down, the hucMSCs were not able to efficiently promote the expression of FoxO3a (Fig. 6a), GLUT1, and GCK (Fig. 6b) and increase glucose uptake (Fig. 6c). At the same time, the improvement of mitochondrial OXPHOS and ATP production were partially weakened in FoxO3 knockdown cells (Fig. $6 \mathrm{~d}-\mathrm{f}$ ) and was accompanied by restoration of glucagon secretion (Fig. $6 \mathrm{~g}-\mathrm{i}$ ). These findings suggest that FoxO3a participates in the effects of hucMSCs on a-cells.

\section{hucMSCs improve hyperglycemia and hyperglucagonemia in vivo via SIRT1}

To further illustrate the role of hucMSCs in vivo through SIRT1, we injected EX-527 into T2DM mice, together with hucMSCs. Our results showed that when co-treated with EX-527, the effects of hucMSCs on improving glucose tolerance and insulin tolerance were obviously weakened (Fig. 7a, b). Meanwhile, circulatory glucagon levels in hucMSCs and the EX-527-treated group were higher than the hucMSCtreated group, while insulin levels were lower (Fig. 7c, d). Additionally, immunofluorescence staining showed that when co-treated with EX-527, hucMSCs did not effectively rescue islet structure and $\alpha$ - and $\beta$ cell imbalance (Fig. 7e-g). Taken together, these results indicate that hucMSCs mitigate hyperglycemia and hyperglucagonemia in vivo via SIRT1.

\section{Discussion}

In this study, we explored the underlying mechanisms by which hucMSCs alleviated pancreatic a-cell dysfunction in T2DM. By activating SIRT1/Fox03a signaling, hucMSCs increased glucose uptake and 
activation, enhanced mitochondrial OXPHOS and promoted ATP production, which eventually reduced glucagon secretion and mitigated hyperglucagonemia in T2DM.

T2DM is characterized by pancreatic $\beta$-cell dysfunction and insulin resistance. a-Cells also play a vital role in maintaining glucose homeostasis by secreting glucagon [1]. T2DM is associated with higher glucagon-to-insulin ratios and augmentation of the $\alpha$ - to $\beta$-cell area ratios, which result in hyperglucagonemia and hyperglycemia $[2,25,26]$. In addition, enhanced glucagon concentrations are also observed in the prediabetes population [27]. MSC-based therapy has been considered as one of the most promising and effective approaches for treating diabetes [12]. There are multiple sources for MSCs acquisition such as bone marrow, adipose tissue, and umbilical cord [28, 29]. hucMSCs can be isolated at ease with fewer ethical issues and have higher proliferative potential and lower immunogenicity compared with other MSCs, which makes hucMSCs an ideal choice for diabetes treatment $[13,14]$. Our previous studies showed that MSCs or their exosomes not only reverse $\beta$-cell dedifferentiation [30] and ameliorate islet endothelium apoptosis [31], but also improve hepatic glucose and lipid metabolism [32]. However, studies showing the role of MSCs in pancreatic a-cell dysfunction are few. In this study, we found that both in HFD and STZ-induced T2DM mice and db/db mice, hucMSC infusion could rescue islet structure and decrease $a$ - to $\beta$-cell ratio, which contribute to amelioration of hyperglycemia and hyperglucagonemia. Consistently, in aTC1-6 cells, hucMSC intervention decreased glucagon levels in the supernatant, whereas upregulated intracellular glucagon protein levels, which indicate that glucagon secretion process was inhibited by hucMSCs in vitro.

Glucagon secretion by a-cells is mainly under the control of glucose and insulin [2]. Upon uptake via GLUTs, glucose is phosphorylated by GCK and participates in mitochondrial oxidation, which results in the accumulation of intracellular ATP and closure of $\mathrm{K}_{\text {ATP-channels and voltage-gated calcium channels }}$ (VGGCs), thereby inhibiting glucagon release [20-24]. Therefore, the effects of mitochondrial function and ATP content in regulating glucagon secretion should not be underestimated. Studies have shown that individuals with T2DM incur functional and morphological alterations in the mitochondria of different tissues and organs [33-36]. Segerstolpe et al. analyzed single-cell transcriptomes from cultured pancreatic islets of T2DM donors, which revealed that genes responsible for energy metabolism in the mitochondria are significantly downregulated in a-cells and $\beta$-cells [3]. Pancreatic a-cells extracted from T2DM mice showed a lower density of mitochondria, a less expressed matrix and a lower number of cristae. These deformities in mitochondrial ultrastructure imply decreased efficiency in mitochondrial ATP production, which accounts for dysregulated glucagon secretion in T2DM [4]. In addition, the regulation of mitochondrial function is also involved in the effects of MSCs on target organs [15-17]. Rackham et al. recently found that human adipose MSCs could transfer mitochondria to $\beta$-cells and promote insulin secretory function [18]. However, the role of MSCs in a-cell mitochondrial function remains unclear. In our study, glucose uptake and mitochondrial OXPHOS were impaired in a-cells after PA exposure, which resulted in reduced ATP production. However, hucMSC treatment promoted glucose uptake and activation, alleviated mitochondrial dysfunction, and enhanced intracellular ATP production. This is the 
first study that illustrates the effects of MSCs on a-cell mitochondrial function; however, the findings require confirmation using in vivo experiments.

Sirtuins participate in regulating metabolism through their regulation of inflammation, oxidative stress, and mitochondrial function via multiple mechanisms, which contribute to the improvement of T2DM [5]. Sirtuins include seven family members, among which SIRT1 plays an important role in mitochondrial biogenesis and oxidative metabolism [6, 7]. Loss of SIRT1 in mouse $\beta$-cells impairs glucose disposal due to insufficient insulin secretion. SIRT1 regulates the expression of specific mitochondria-related genes that control metabolic coupling [11]. Bordone et al. found that SIRT1 knockout mice display constitutively high UCP2 expression, which is associated with a failure of cells to increase ATP levels and insulin secretion after glucose stimulation [10]. However, studies illustrating the role of SIRT1 in a-cells are limited. In this study, we first investigated the effects of SIRT1 on glucose uptake and activation, mitochondrial function, and glucagon secretion in a-cells. Moreover, our previous study has suggested that MSC-CM improved mitochondrial function in liver via SIRT1, which is beneficial for diabetesassociated non-alcoholic fatty liver disease (NAFLD) [37]. Meanwhile, other studies showed the effects of MSCs or extracellular vesicles on target tissues via SIRT1 $[17,38]$. Therefore, we explored whether hucMSCs regulate SIRT1 signaling in a-cells. In aTC1-6 cells, the upregulation of SIRT1 signaling and mitochondrial OXPHOS were partially abolished after hucMSC intervention when simultaneously treated with SIRT1 inhibitor EX-527 or when SIRT1 was knocked down, which resulted in restoration of glucagon secretion. Consistently, EX-527 infusion also weakened the effects of hucMSCs on hyperglycemia and hyperglucagonemia in T2DM mice, which indicate that hucMSCs regulate glucagon secretion via SIRT1 signaling.

\section{Conclusions}

In summary, our observations indicate that by activating SIRT1/FoxO3a signaling, hucMSCs could increase glucose uptake and activation, enhance mitochondrial OXPHOS and promote ATP production, which eventually reduce glucagon secretion and mitigate hyperglucagonemia in T2DM. This is first study that shows that hucMSCs alleviate mitochondrial dysfunction and glucagon hypersecretion of a-cells in T2DM, which provides novel evidence demonstrating the potential for hucMSCs in treating T2DM.

\section{Abbreviations}

T2DM:type 2 diabetes mellitus; MSCs:mesenchymal stem cells; hucMSCs:human umbilical cord mesenchymal stem cells; ELISA:enzyme-linked immunosorbent assay; SIRT1: sirtuin 1; FoxO3a: forkhead box O3a; siRNA:small interfering RNA; HFD:high-fat diet; PA:palmitate; FBS:fetal bovine serum; HELF:human embryo lung fibroblasts; DMEM:Dulbecco's modified Eagle's medium; STZ: streptozocin; IPGTT:intraperitoneal glucose tolerance test; IPITT:intraperitoneal insulin tolerance test; AUC:area under the curve; KRBH:Krebs-Ringer bicarbonate HEPES; RIPA:radioimmunoprecipitation assay; BCA:bicinchoninic acid; TEM:transmission electron microscopy; OCR:oxygen consumption rate; 
siRNA:small interfering RNA; GLUT1:glucose transporter type 1; GCK:glucokinase; OXPHOS:oxidative phosphorylation

\section{Declarations}

\section{Acknowledgments}

We thank LetPub (www.letpub.com) for its linguistic assistance during the preparation of this manuscript.

\section{Authors' contributions}

JS performed the experiments, collected data, performed data analysis, and wrote the manuscript. LSW, $\mathrm{XHG}, \mathrm{QH}$, and CC helped perform the experiments. $\mathrm{HQH}, \mathrm{NZ}$, and MMY helped with the sample collection. $\mathrm{FY}, \mathrm{KL}, \mathrm{CW}, \mathrm{FQL}, \mathrm{YJS}$ and FLH provided technical support and guided the data analysis. ZS, XGH, HL and LC supervised the overall study design. All authors read and approved the final version of the manuscript.

\section{Funding}

This work was supported by the National Natural Science Foundation of China (grant numbers 81873632, 82070800, 81770818, 81800727, 81900756), the Taishan Scholar Foundation of Shandong Province (ts201712089), the Key Research and Development Program of Shandong Province(2019GSF108099), the Natural Science Foundation of Shandong Province (ZR2019BH018, ZR2019PH078), and the Taishan Industry Leading Talent Project of Shandong Province.

\section{Availability of data and materials}

All data generated or analysed during this study are included in this published article.

\section{Ethics approval and consent to participate}

All animal experiments were approved by the Animal Ethics Committee of Shandong University(Ethics Number:DWLL-2019-016).

\section{Competing interests}

All authors declare that they have no competing interests. 


\section{Author details}

${ }^{1}$ Department of Endocrinology, Qilu Hospital, Cheeloo College of Medicine, Shandong University, No.107 Wenhua Xi Road, Jinan 250012, Shandong, China. ${ }^{2}$ Institute of Endocrine and Metabolic Diseases of Shandong University, Jinan 250012, Shandong, China. ${ }^{3}$ Key Laboratory of Endocrine and Metabolic Diseases, Shandong Province Medicine \& Health, Jinan 250012, Shandong, China. ${ }^{4}$ Jinan Clinical Research Center for Endocrine and Metabolic Disease, Jinan, 250012, Shandong, China. ${ }^{5}$ Nuolai Biomedical Technology Co., Ltd., Taian, 271001, Shandong, China.

\section{References}

1. Baetens D, Malaisse-Lagae F, Perrelet A, et al. Endocrine pancreas:three-dimensional reconstruction showstwotypes of islets of langerhans. Science. 1979;206:1323-5. doi:10.1126/science.390711.

2. Dunning BE, Gerich JE. The role of alpha-cell dysregulation in fasting and postprandial hyperglycemia in type 2 diabetes and therapeutic implications. Endocr Rev. 2007;28:253-83. doi:10.1210/er.2006-0026.

3. Segerstolpe Å, Palasantza A, Eliasson P, et al. Single-Cell Transcriptome Profiling of Human Pancreatic Islets in Health and Type 2 Diabetes. Cell Metab. 2016;24(4):593-607. doi:10.1016/j.cmet.2016.08.020.

4. Grubelnik V, Markovič R, Lipovšek S,et al. Modelling of dysregulated glucagon secretion in type 2 diabetes by considering mitochondrial alterations in pancreatic a-cells. R Soc Open Sci. 2020;7(1):191171. doi:10.1098/rsos.191171.

5. Kitada M, Ogura Y, Monno I, et al. Sirtuins and Type 2 Diabetes: Role in Inflammation, Oxidative Stress, and Mitochondrial Function. Front Endocrinol (Lausanne). 2019;10:187. doi:10.3389/fendo.2019.00187.

6. Canto $C$, Gerhart-Hines Z, Feige JN,et al. AMPK regulates energy expenditure by modulating NAD+ metabolism and SIRT1 activity. Nature. 2009;458:1056-60. doi:10.1038/nature07813.

7. Price NL, Gomes AP, Ling AJ, et al. SIRT1 is required for AMPK activation and the beneficial effects of resveratrol on mitochondrial function. Cell Metab. 2012;15:675-90. doi:10.1016/j.cmet.2012.04.003.

8. Hsu YH, Chen YC, Chen YW, et al. Far-infrared radiation prevents decline in $\beta$-cell mass and function in diabetic mice via the mitochondria-mediated Sirtuin1 pathway. Metabolism. 2020;104:154143. doi:10.1016/j.metabol.2020.154143.

9. Moynihan KA, Grimm AA, Plueger MM, et al. Increased dosage of mammalian Sir2 in pancreatic beta cells enhances glucose-stimulated insulin secretion in mice. Cell Metab. 2005;2:105-17. doi:10.1016/j.cmet.2005.07.001. 
10. Bordone L, Motta MC, Picard F, et al. Sirt1 regulates insulin secretion by repressing UCP2 in pancreatic beta cells. PLoS Biol. 2006;4:e31. doi:10.1371/journal.pbio.0040031.

11. Luu L, Dai FF, Prentice KJ, et al. The loss of Sirt1 in mouse pancreatic beta cells impairs insulin secretion by disrupting glucose sensing. Diabetologia. 2013;56:2010-20. doi:10.1007/s00125-0132946-5.

12. Xv J, Ming Q, Wang X, et al. Mesenchymal stem cells moderate immune response of type 1 diabetes. Cell Tissue Res. 2017;368:239-48. doi:10.1007/s00441-016-2499-2.

13. Chandravanshi $B$, Bhonde RR. Human umbilical cord-derived stem cells: isolation, characterization, differentiation, and application in treating diabetes. Crit Rev Biomed Eng. 2018;46:399-412. doi:10.1615/CritRevBiomedEng.2018027377.

14. Xie Z, Hao H, Tong C, et al. Human umbilical cord derived mesenchymal stem cells elicit macrophages into an anti inflammatory phenotype to alleviate insulin resistance in type 2 diabetic rats. Stem Cells. 2016;34:627-39. doi:10.1002/stem.2238.

15. Liu X, Li X, Zhu W, et al. Exosomes from mesenchymal stem cells overexpressing MIF enhance myocardial repair. J Cell Physiol. 2020;235(11):8010-22. doi:10.1002/jcp.29456.

16. Hughey CC, James FD, Ma L, et al. Diminishing impairments in glucose uptake, mitochondrial content, and ADP-stimulated oxygen flux by mesenchymal stem cell therapy in the infarcted heart. Am J Physiol Cell Physiol. 2014;306(1):C19-27. doi:10.1152/ajpcell.00156.2013.

17. Yuan Y, Shi M, Li L, et al. Mesenchymal stem cell-conditioned media ameliorate diabetic endothelial dysfunction by improving mitochondrial bioenergetics via the Sirt1/AMPK/PGC-1a pathway. Clin Sci (Lond). 2016;130(23):2181-98. doi:10.1042/CS20160235.

18. Rackham CL, Hubber EL, Czajka A,et al. Optimizing beta cell function through mesenchymal stromal cell-mediated mitochondria transfer. Stem Cells. 2020 Apr;38(4):574-584. doi: 10.1002/stem.3134.

19. Song J, He Q, Guo X, et al. Mesenchymal stem cell-conditioned medium alleviates high fat-induced hyperglucagonemia via miR-181a-5p and its target PTEN/AKT signaling. Mol Cell Endocrinol. 2021;537:111445. doi:10.1016/j.mce.2021.111445.

20. Rorsman P, Salehi SA, Abdulkader F, et al. K(ATP)-channels and glucose-regulated glucagon secretion. Trends Endocrinol Metab. 2008;19:277-84. doi:10.1016/j.tem.2008.07.003.

21. Leung YM, Ahmed I, Sheu L, et al. Insulin regulates islet a-cell function by reducing $\mathrm{K}_{\text {ATP }}$ channel sensitivity to adenosine 5-triphosphate inhibition. Endocrinology. 2006;147:2155-62. doi:10.1210/en.2005-1249.

22. Khan FA, Goforth PB, Zhang M, et al. Insulin activates ATP-sensitive $\mathrm{K}(+)$ channels in pancreatic acells through a phosphatidylinositol 3-kinase-dependent pathway. Diabetes. 2001;50:2192-8. doi:10.2337/diabetes.50.10.2192.

23. Berger C, Zdzieblo D. Glucose transporters in pancreatic islets. Pflugers Arch. 2020;472(9):1249-72. doi:10.1007/s00424-020-02383-4. 
24. Basco D, Zhang Q, Salehi A,et al. a-cell glucokinase suppresses glucose-regulated glucagon secretion. Nat Commun. 2018;9(1):546. doi:10.1038/s41467-018-03034-0.

25. Fujita $Y$, Kozawa J, Iwahashi $\mathrm{H}$, et al. Human pancreatic alpha- to beta-cell area ratio increases after type 2 diabetes onset. J Diabetes Investig. 2018;9:1270-82. doi:10.1111/jdi.12841.

26. Lee $M$, Kim M, Park JS, et al. Higher glucagon-to-insulin ratio is associated with elevated glycated hemoglobin levels in type 2 diabetes patients. Korean J Intern Med. 2019;34(5):1068-77. doi:10.3904/kjim.2016.233.

27. Sharma A, Varghese RT, Shah M, et al. Impaired Insulin Action Is Associated With Increased Glucagon Concentrations in Nondiabetic Humans. J Clin Endocrinol Metab. 2018;103(1):314-9. doi:10.1210/jc.2017-01197.

28. Shi H, Xu X, Zhang B, et al. 3,3'-Diindolylmethane stimulates exosomal Wnt11 autocrine signaling in human umbilical cord mesenchymal stem cells to enhance wound healing. Theranostics. 2017;7:1674-88. doi:10.7150/thno.18082.

29. Si Y, Zhao Y, Hao H, et al. Infusion of mesenchymal stem cells ameliorates hyperglycemia in type 2 diabetic rats: identification of a novel role in improving insulin sensitivity. Diabetes. 2012;61:161625. doi:10.2337/db11-1141.

30. He Q, Song J, Cui C, et al. Mesenchymal stem cell-derived exosomal miR-146a reverses diabetic $\beta$-cell dedifferentiation. Stem Cell Res Ther. 2021;12(1):449. doi:10.1186/s13287-021-02371-0.

31. Wang L, Qing L, Liu H, et al. Mesenchymal stromal cells ameliorate oxidative stress-induced islet endothelium apoptosis and functional impairment via Wnt4-beta-catenin signaling. Stem Cell Res Ther. 2017;8:188. doi:10.1186/s13287-017-0640-0.

32. He Q, Wang L, Zhao R, et al. Mesenchymal stem cell-derived exosomes exert ameliorative effects in type 2 diabetes by improving hepatic glucose and lipid metabolism via enhancing autophagy. Stem Cell Res Ther. 2020 Jun 8;11(1):223. doi: 10.1186/s13287-020-01731-6.

33. Anello M. Lupi R, Spampinato $D$, et al Functional and morphological alterations of mitochondria in pancreatic beta cells from type 2 diabetic patients. Diabetologia. 2005;48 (2):282-9. doi: 10.1007/s00125-004-1627-9.

34. Kelley DE. He J, Menshikova EV, et al Dysfunction of mitochondria in human skeletal muscle in type 2 diabetes. Diabetes. 2002;51 (10): 2944-50. doi: 10.2337/diabetes.51.10.2944.

35. Heinonen S. Buzkova J, Muniandy $\mathrm{M}$, et al Impaired mitochondrial biogenesis in adipose tissue in acquired obesity. Diabetes. 2015;64 (9):3135-45. doi: 10.2337/db14-1937.

36. Pisano A. Cerbelli B, Perli E,et al. Impaired mitochondrial biogenesis is a common feature to myocardial hypertrophy and end-stage ischemic heart failure. Cardiovasc Pathol 2016;25 (2):10312. doi: 10.1016/j.carpath.2015.09.009.

37. Yang M. Cui Y, Song J, et al Mesenchymal stem cell-conditioned medium improved mitochondrial function and alleviated inflammation and apoptosis in non-alcoholic fatty liver disease by regulating SIRT1. Biochem Biophys Res Commun. 2021;546:74-82. doi: 10.1016/j.bbrc.2021.01.098. 
38. Feng R. Ullah M, Chen $\mathrm{K}$, et al Stem cell-derived extracellular vesicles mitigate ageing-associated arterial stiffness and hypertension. J Extracell Vesicles. 2020;9(1):1783869. doi: 10.1080/20013078.2020.1783869.

\section{Figures}
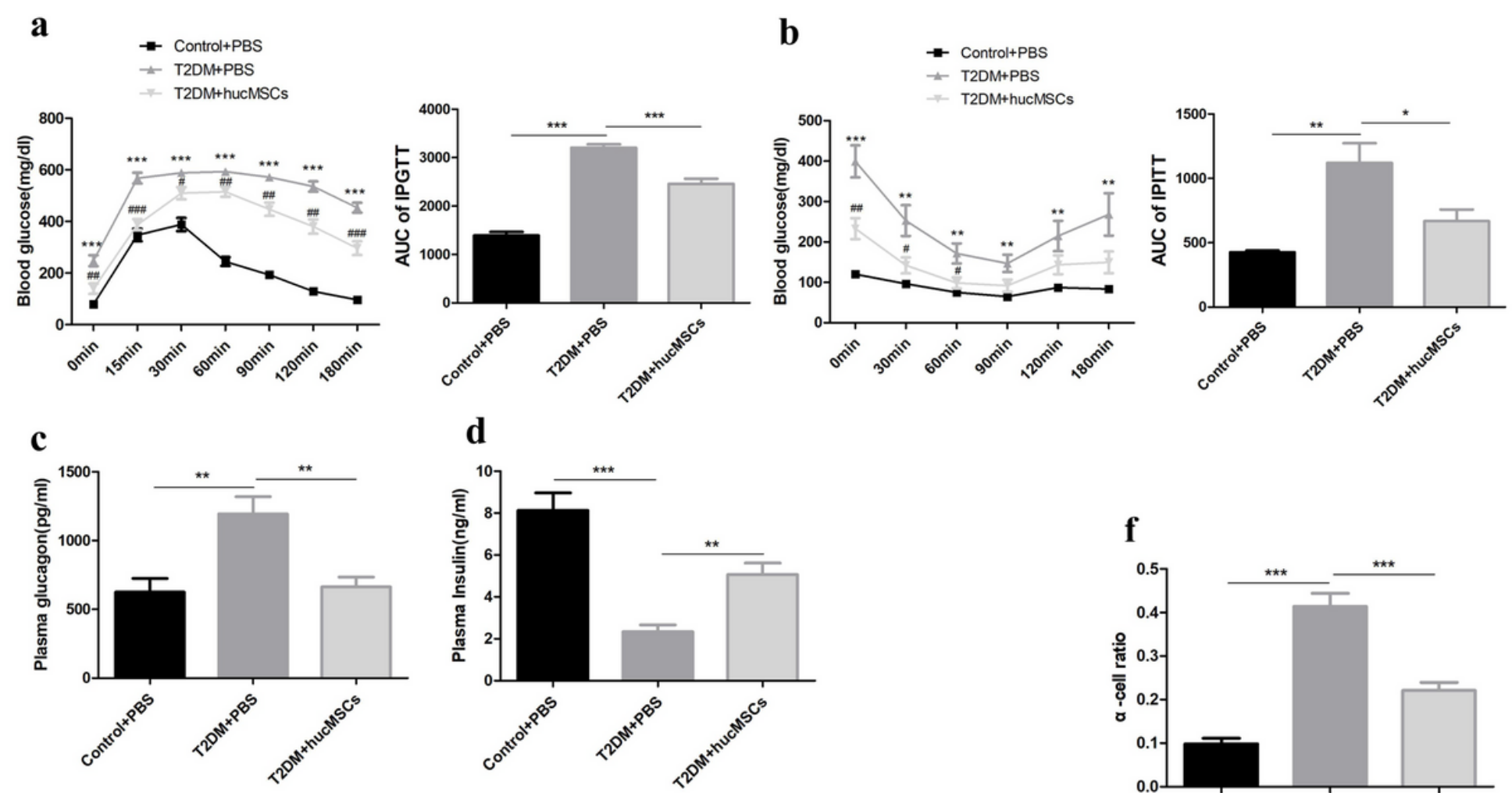

d
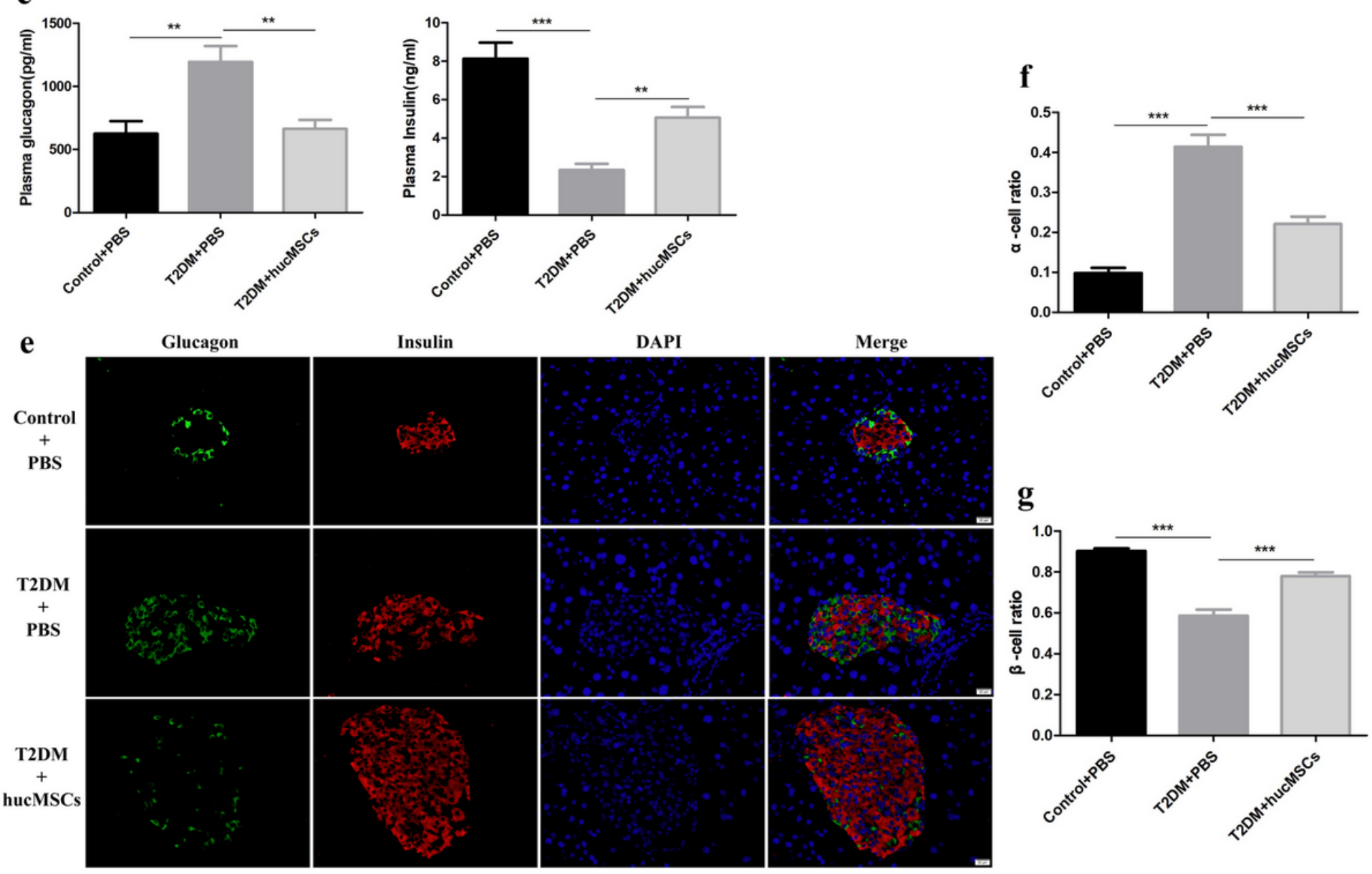

\section{Figure 1}

hucMSC infusion ameliorates hyperglycemia and hyperglucagonemia in HFD- and STZ-induced T2DM mice. a. IPGTT showed that hucMSC infusion reduced fasting glucose levels and improved glucose 
tolerance. The AUC supported the above findings ( $\mathrm{n}=6$ mice per group). b. IPGTT showed that hucMSC infusion improved insulin tolerance. c. hucMSC intervention decreased circulatory glucagon levels in T2DM mice. d. hucMSC intervention increased circulatory insulin levels in T2DM mice. e.

Immunofluorescence staining showed that hucMSC administration partially rescued islet structure. $\mathrm{f}, \mathrm{g}$. hucMSC infusion reduced a-cell ratio and elevated $\beta$-cell ratio, as analyzed by Image-Pro Plus software ( $\mathrm{n}$

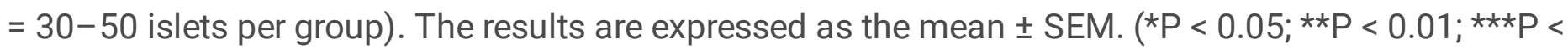
$0.001)$.
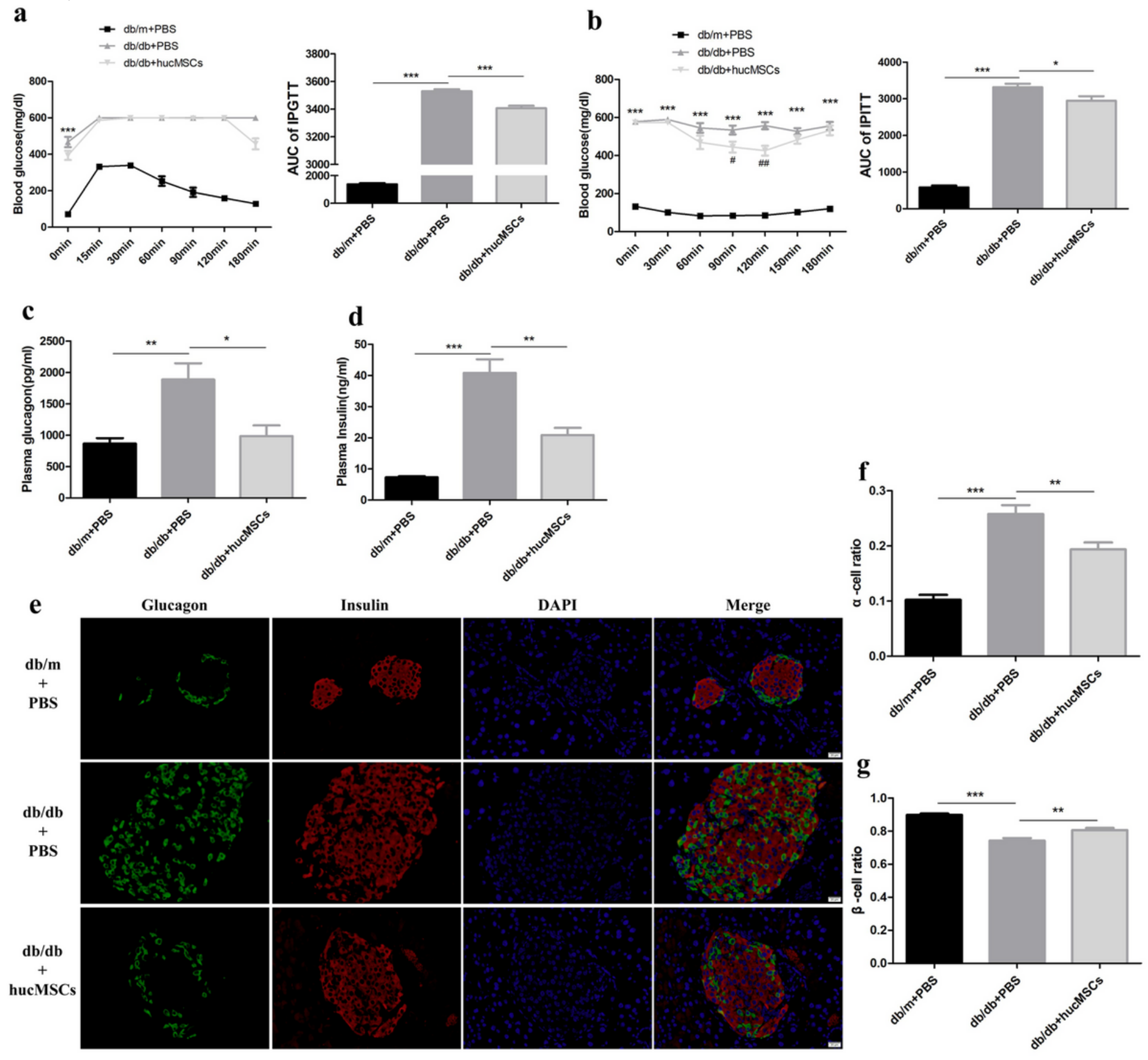

Figure 2 
hucMSC infusion alleviates hyperglycemia and hyperglucagonemia in $\mathrm{db} / \mathrm{db}$ mice. a. IPGTT showed that hucMSC infusion reduced fasting glucose levels and improved glucose tolerance. The AUC supported the above findings ( $n=6$ mice per group). b. IPITT shows that hucMSC infusion improved insulin tolerance. $c, d$. hucMSC intervention decreased circulatory glucagon and insulin levels in $\mathrm{db} / \mathrm{db}$ mice. e. Immunofluorescence staining showed that hucMSC administration partially rescued islet structure. $\mathrm{f}, \mathrm{g}$. hucMSC infusion reduced $\alpha$-cell ratio and elevated $\beta$-cell ratio, as analyzed by Image-Pro Plus software ( $\mathrm{n}$ $=50-80$ islets per group). The results are expressed as the mean \pm SEM $(* P<0.05 ; * * P<0.01 ; * \star * P<$ $0.001)$.

a

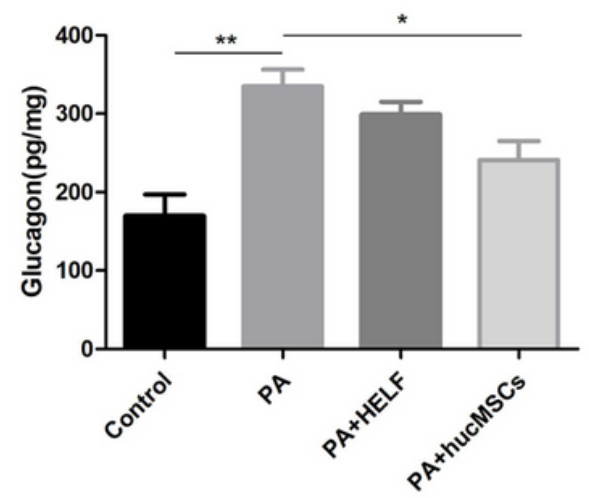

b

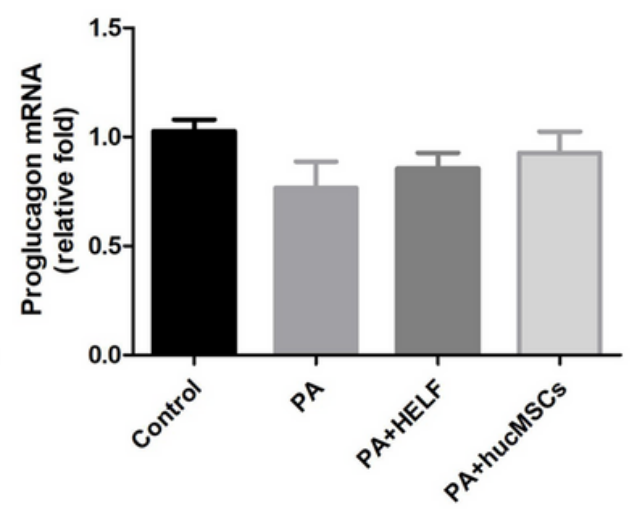

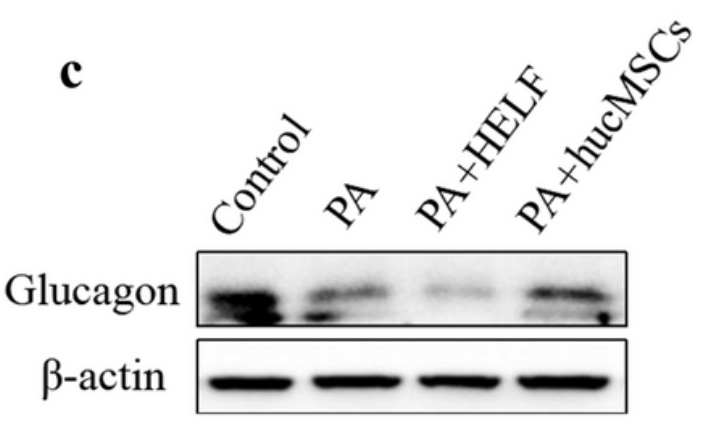

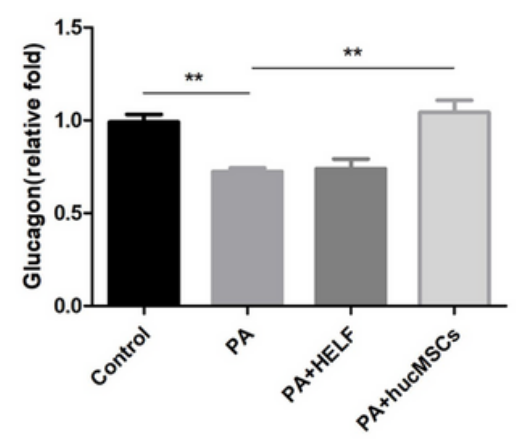

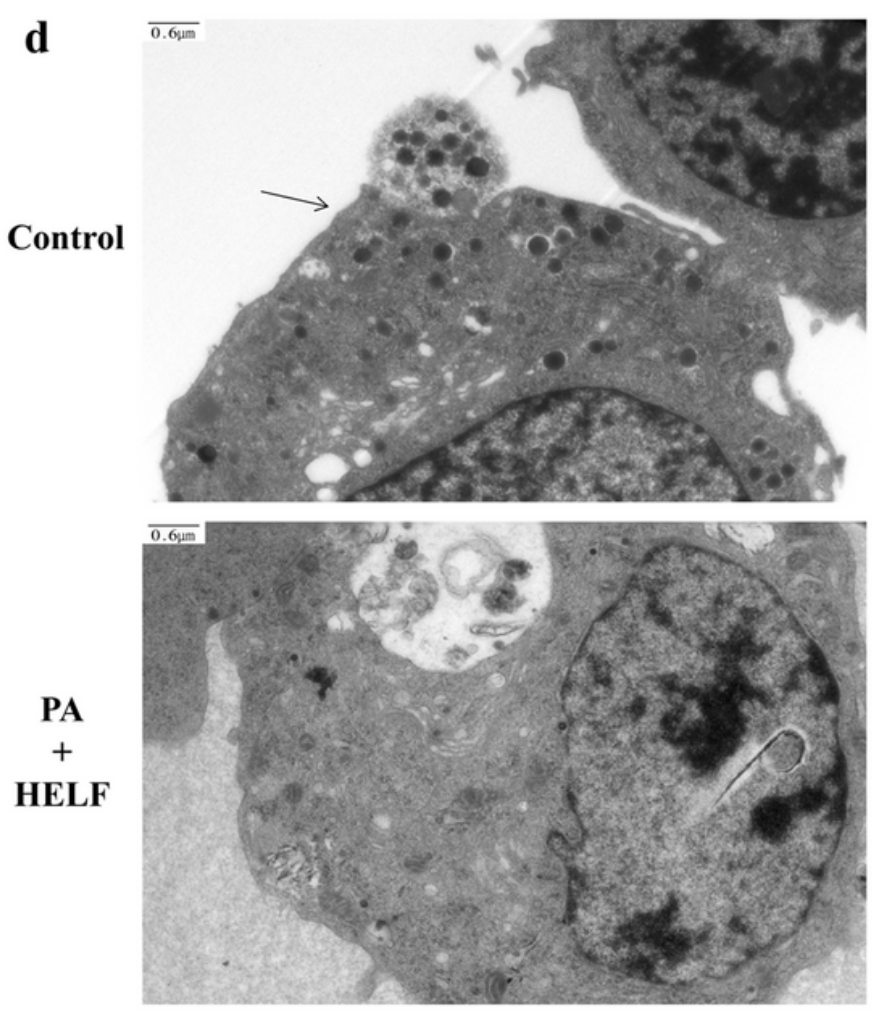

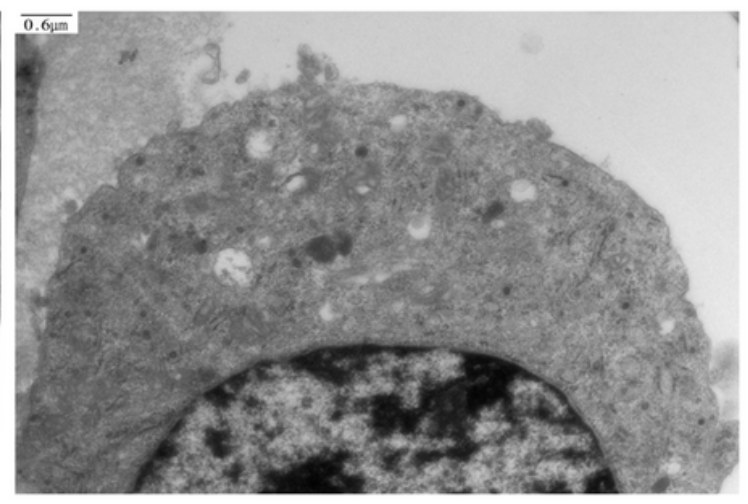

PA

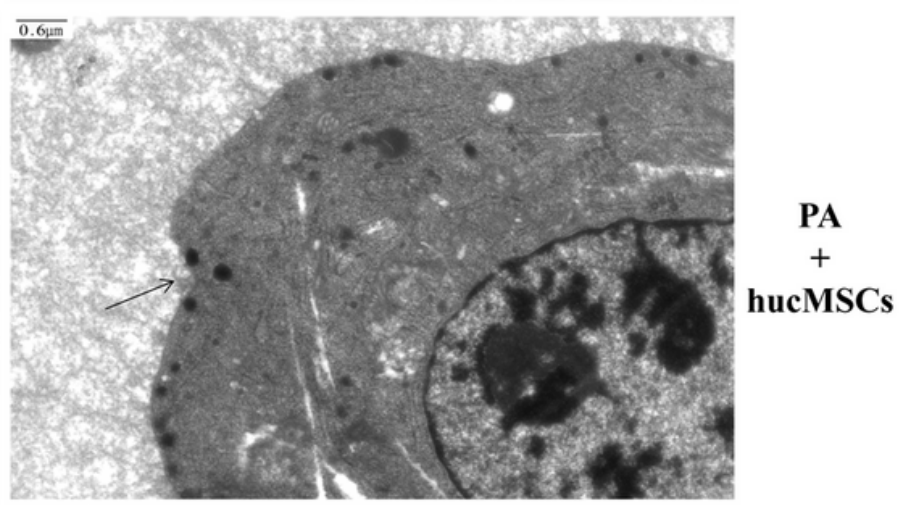

Figure 3 
hucMSCs decrease glucagon secretion in aTC1-6 cells. a. ELISA showed that hucMSC treatment remarkably reduced glucagon levels in the supernatant. b. No significant changes in proglucagon mRNA levels were observed, which was confirmed by RT-qPCR. c. Western blot demonstrated that hucMSCs promoted intracellular glucagon expression. Quantification of bands was performed using ImageJ software. d. TEM images showed fewer glucagon granules in the PA group, whereas more granules were observed in the hucMSC group, which were concordant to intracellular glucagon protein levels. All these experiments were repeated three to four times, and the results were presented as the mean \pm SEM $(* P<$ $0.05 ; * \star P<0.01)$. 

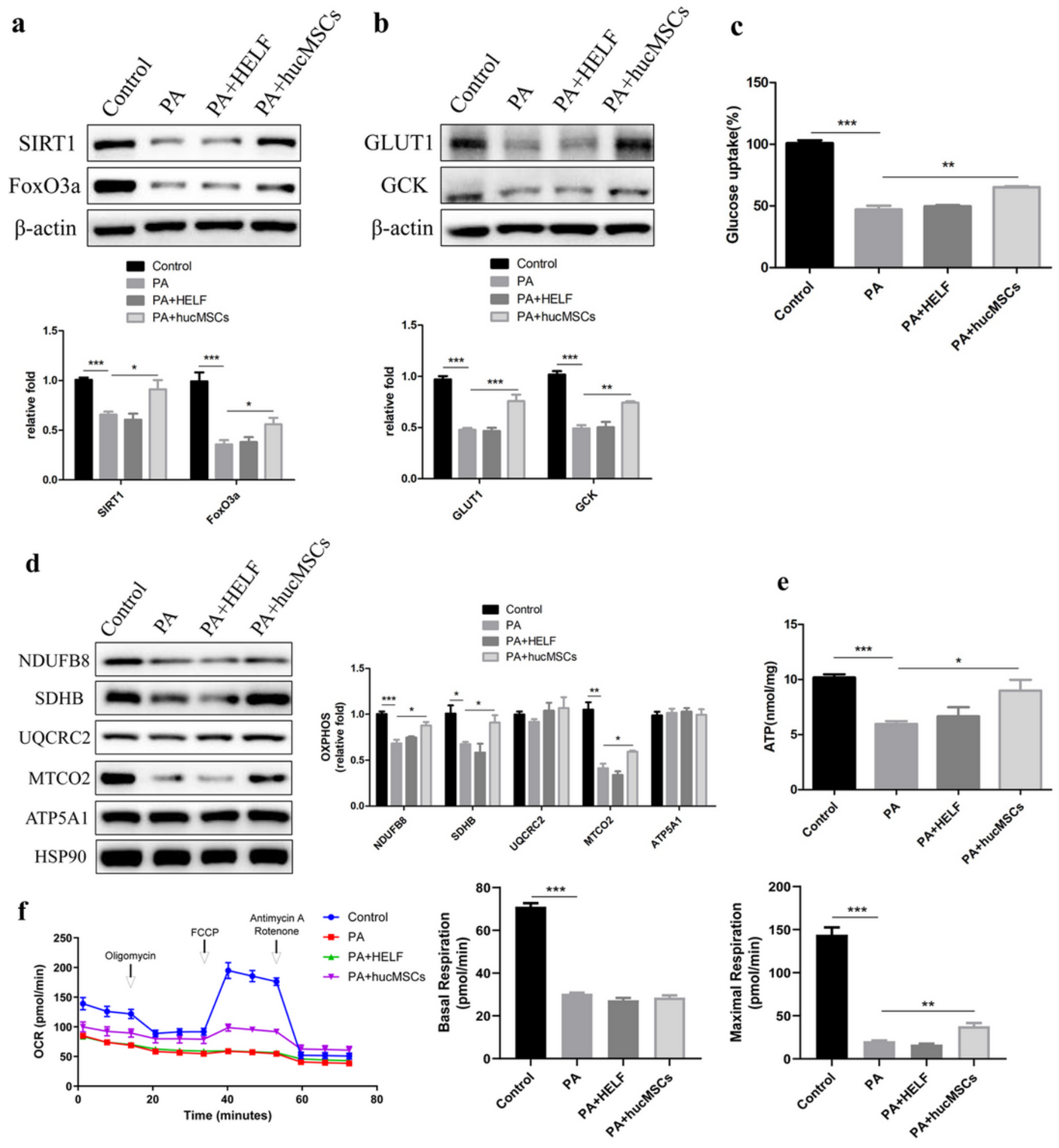

Figure 4

hucMSCs regulate SIRT1/FoxO3a signaling and improve mitochondrial function in aTC1-6 cells. a. Western blot showed that PA downregulated the expression of SIRT1 and its downstream FoxO3a, while hucMSCs promoted SIRT1/Fox03a signaling. Quantification of bands was performed using ImageJ software. b. hucMSCs upregulated glucose transporter GLUT1 and glucokinase (GCK) levels. c. hucMSCs increased glucose uptake, as detected by fluorescent glucose 2-NBDG. d. Western blotting indicated that 
hucMSC therapy elevated expression of mitochondrial complex NDUFB8, SDHB, and MTCO2. e. hucMSC treatment promoted ATP production in a-cells. f. Seahorse analysis showed that hucMSCs enhanced mitochondrial OXPHOS, as evidenced by increased maximal respiration. All of these experiments were repeated three to four times, and the data were presented as the mean \pm SEM $\left({ }^{\star} P<0.05 ;{ }^{* \star P} P<0.01 ; * \star \star P<\right.$ $0.001)$.
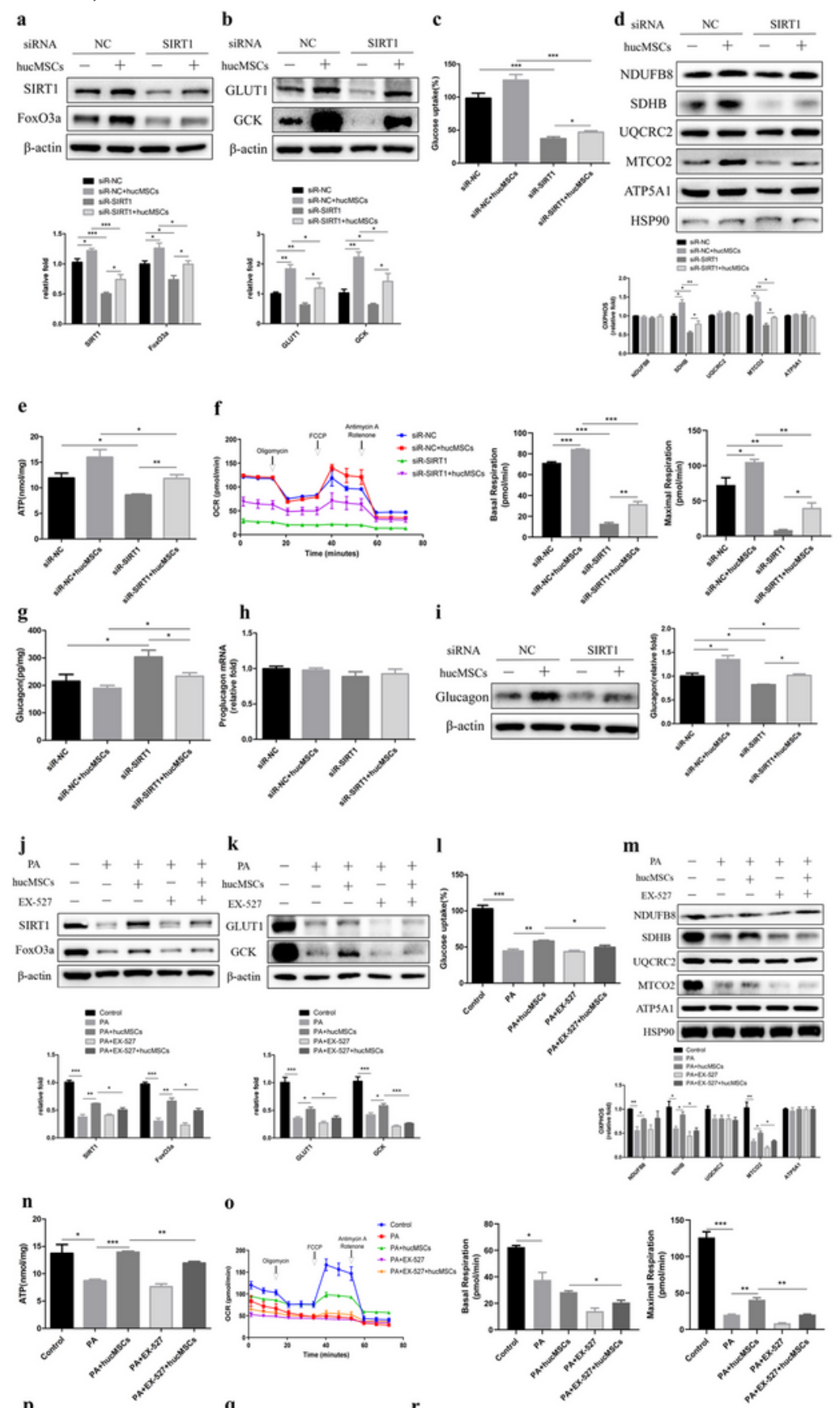

o
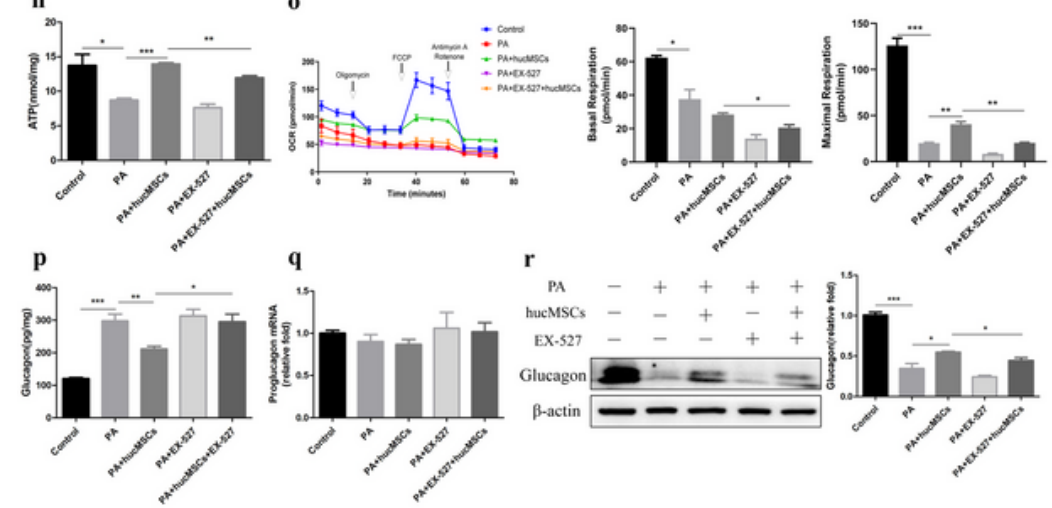

Figure 5 
hucMSCs mitigate mitochondrial dysfunction and glucagon hypersecretion by activating SIRT1. a. Western blotting showed that in SIRT1 knockdown cells, hucMSC treatment weakly increased SIRT1 and FoxO3a expression. Quantification of bands was performed using ImageJ software. b, c. Effects of hucMSCs on the upregulation of GLUT1, GCK, and glucose uptake were partially blocked when SIRT1 was knocked down. d. Effects of hucMSCs on elevation of mitochondrial complex SDHB and MTCO2 were partially blocked when SIRT1 was knocked down. e. hucMSC therapy did not sufficiently restore ATP production in SIRT1 knockdown cells. $\mathrm{f}$. Seahorse analysis showed that the role of hucMSCs in enhancing mitochondrial OXPHOS was partially weakened in SIRT1 knockdown cells, as evidenced by basal respiration and maximal respiration. $\mathrm{g}$. ELISA showed that glucagon secretion was not effectively inhibited by hucMSCs when SIRT1 was knocked down. h. RT-qPCR suggested there were no significant changes in proglucagon mRNA levels. i. Western blotting showed that intracellular glucagon protein levels were not effectively elevated by hucMSCs. j. Upregulation of SIRT1 and FoxO3a was weakened after hucMSC therapy when simultaneously treated with SIRT1 inhibitor EX-527. k,l. hucMSCs weakly elevated GLUT1 and GCK expression and glucose uptake when co-treated with EX-527. m-o. Improvement of mitochondrial OXPHOS and ATP production was also weakened in hucMSCs and EX-527 co-treated group compared with hucMSCs-treated group. p. ELISA showed that the role of hucMSCs in decreasing glucagon secretion was blocked when co-treated with EX-527. q. No significant changes in proglucagon mRNA levels were observed, which was confirmed by RT-qPCR. r. Western blotting indicated that the effects of hucMSCs on elevation of intracellular glucagon levels were partially blocked when co-treated with EX-527. All of the experiments were repeated three to four times, and the data were presented as the mean \pm SEM $(* \mathrm{P}<0.05 ; * \star \mathrm{P}<0.01 ; * \star * \mathrm{P}<0.001)$. 


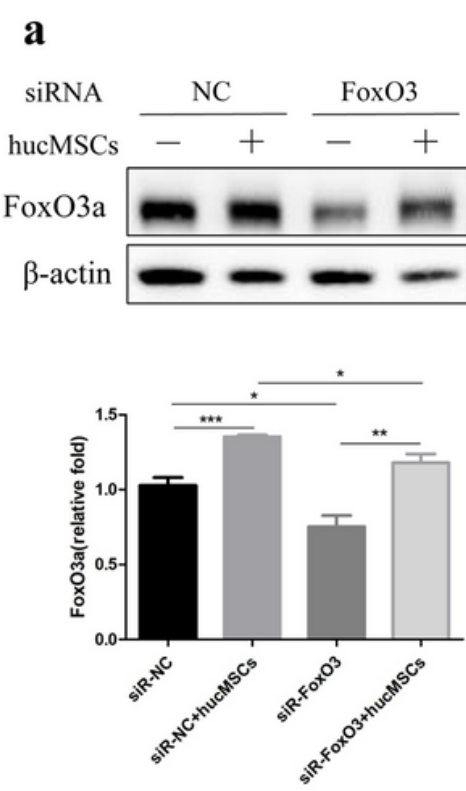

b

$\begin{array}{llll}\text { b }_{\text {SiRNA }} \quad \mathrm{NC} & \mathrm{FoxO}_{3} \quad \mathbf{c}\end{array}$

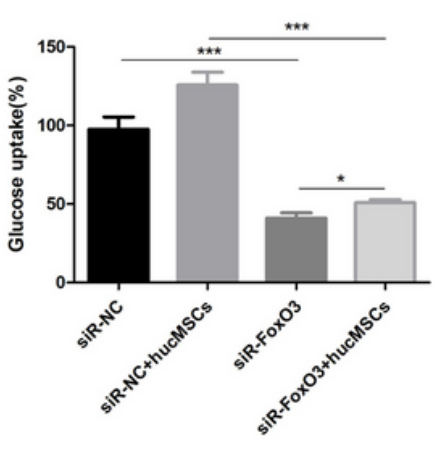

d

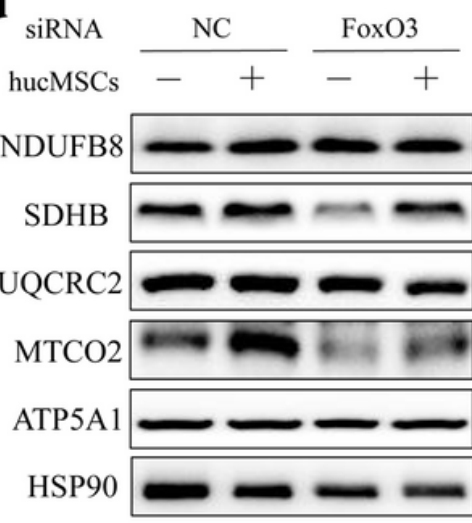

- siR-NC

siR-NC+hucMSCs

sir-FoxOS

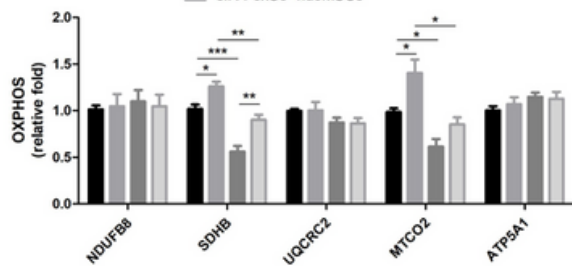

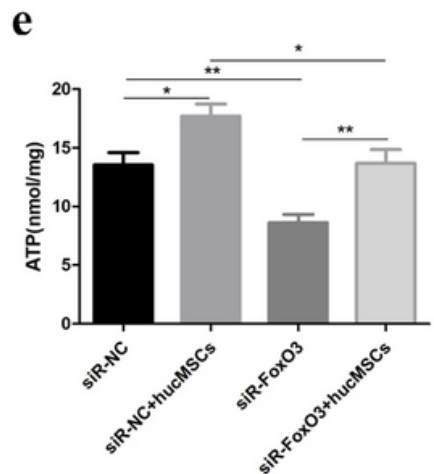

g

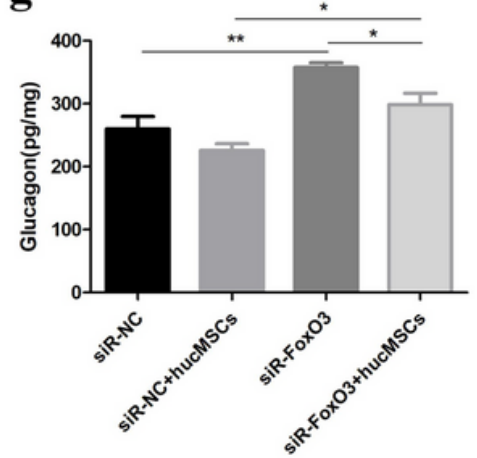

f

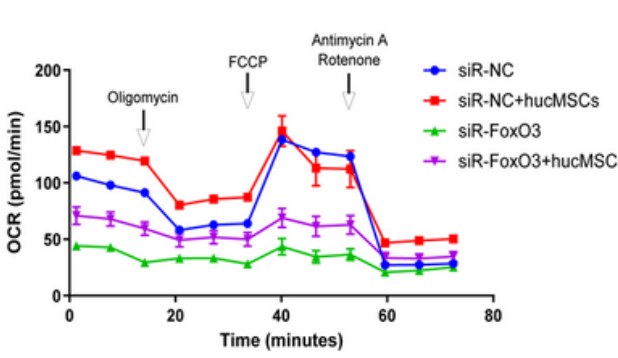

i
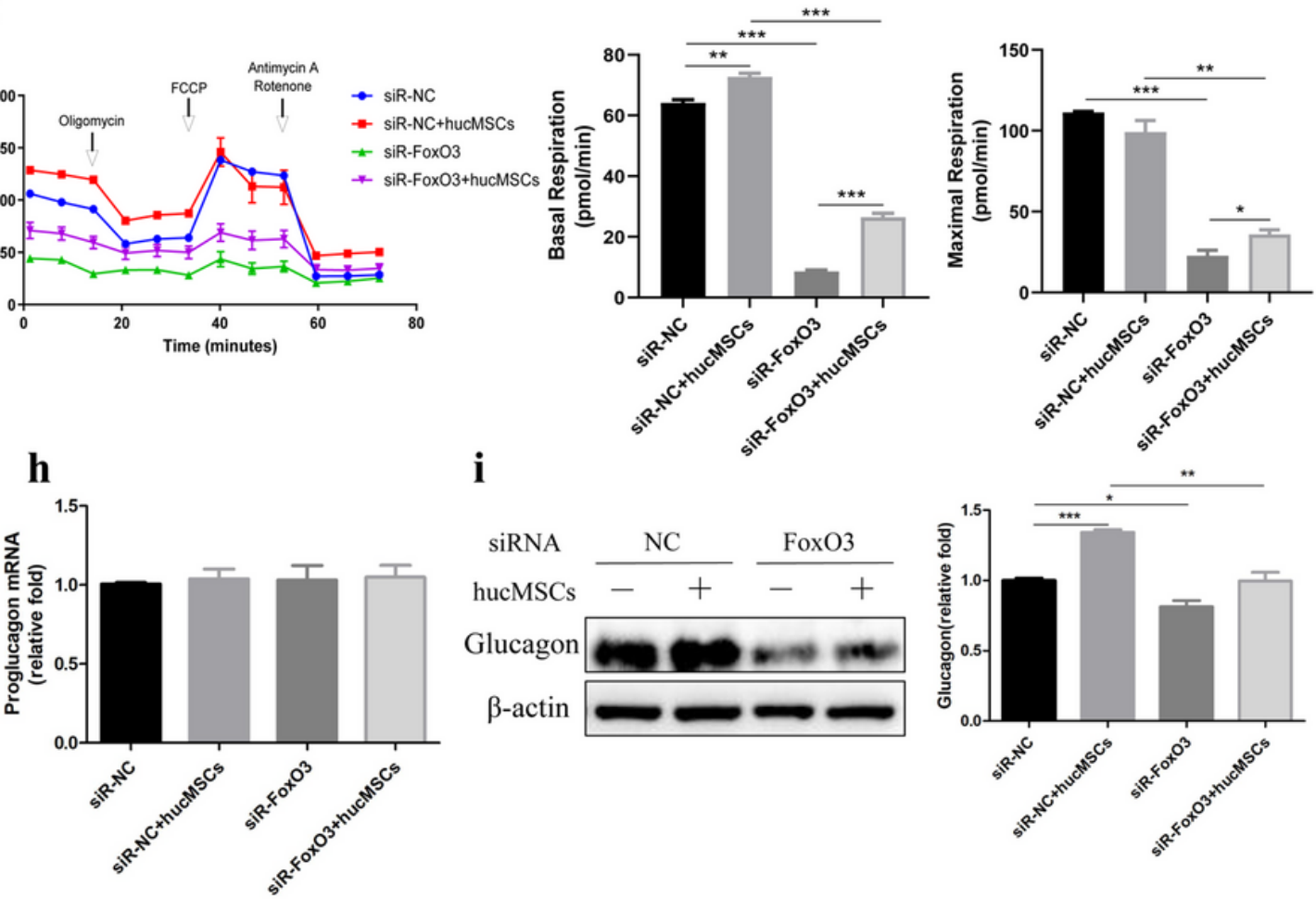

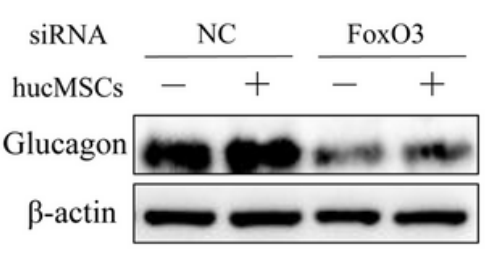

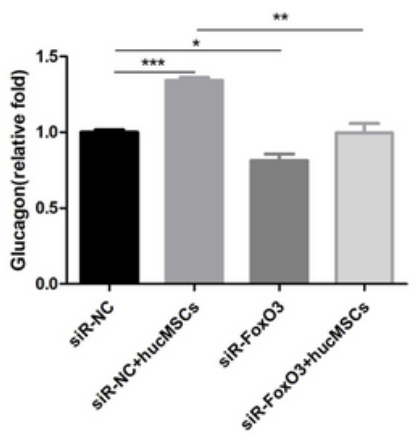

Figure 6

FoxO3a is involved in the improvement of mitochondrial dysfunction and glucagon hypersecretion by hucMSCs. a. aTC1-6 cells were transfected with FoxO3 siRNA. Western blotting showed that when FoxO3 was knocked down, hucMSCs did not efficiently promote FoxO3a expression. Quantification of bands was performed using ImageJ software. b, c. The effects of hucMSCs on the upregulation of GLUT1, GCK, and glucose uptake were partially blocked when $\mathrm{FoxO} 3$ was knocked down. $\mathrm{d}$. Western blotting indicated that the effects of hucMSCs on enhancing mitochondrial complex SDHB and MTCO2 expression were partially blocked when FoxO3 was knocked down. e. The improvement of ATP production was partially 
weakened in Fox03 knockdown cells. f. Seahorse analysis showed that the role of hucMSCs in enhancing mitochondrial OXPHOS was partially blocked in FoxO3 knockdown cells, as evidenced by basal respiration and maximal respiration. g. ELISA showed that glucagon secretion was not effectively inhibited by hucMSCs when FoxO3 was knocked down. h. No significant changes in proglucagon mRNA level were observed, as confirmed by RT-qPCR. i. Western blotting showed that intracellular glucagon protein levels were not efficiently elevated by hucMSCs. All experiments were repeated three to four times, and the data were presented as the mean \pm SEM $(* P<0.05 ; * * P<0.01 ; * * * P<0.001)$.
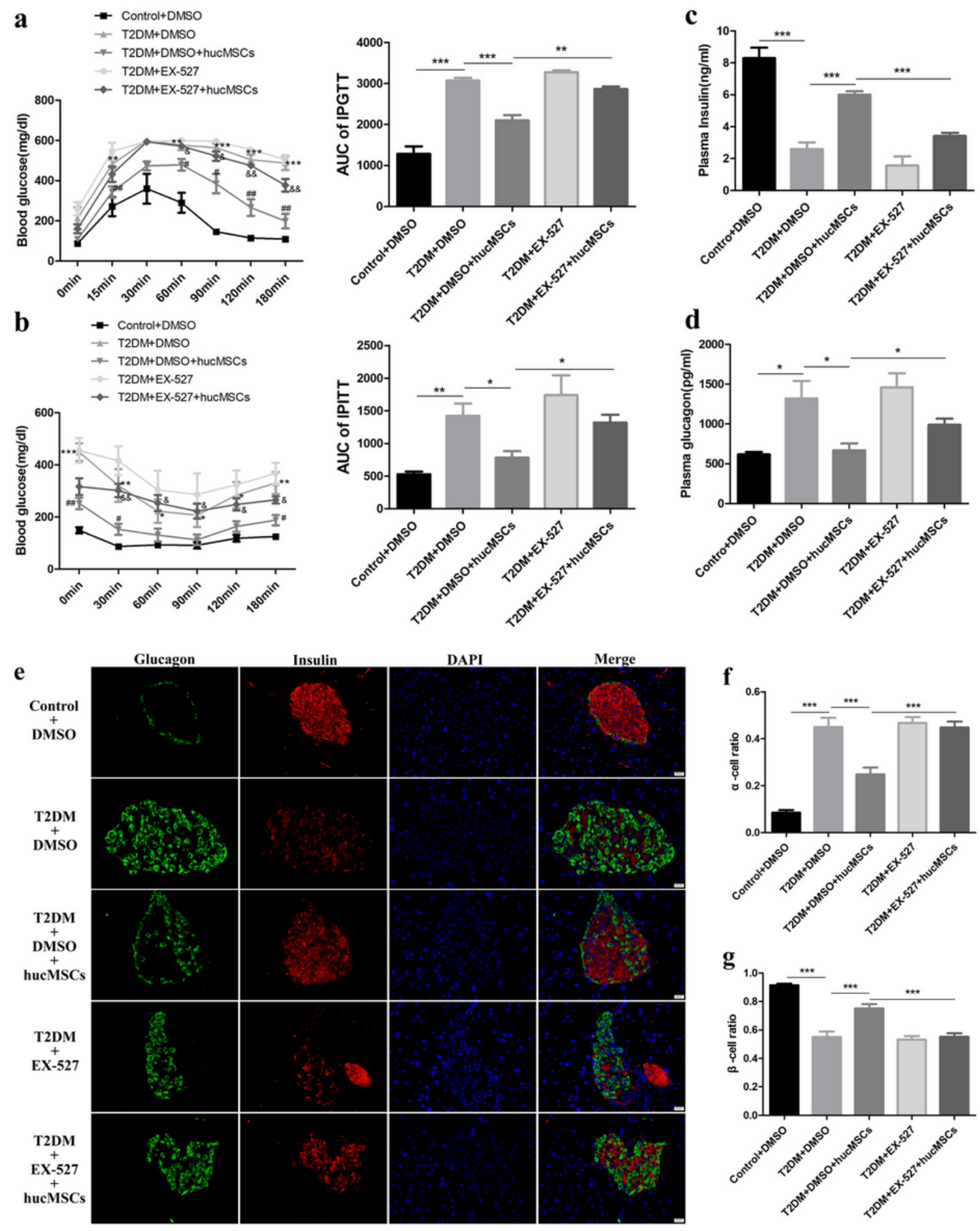


\section{Figure 7}

hucMSCs improve hyperglycemia and hyperglucagonemia in vivo via SIRT1. a. HFD and STZ-induced T2DM mice were simultaneously injected with hucMSCs and EX-527. IPGTT showed that the effects of hucMSCs on improving glucose tolerance and insulin tolerance were obviously weakened. The AUC supported the above findings ( $n=4$ mice per group). b. The results of IPITT showed that the effects of hucMSCs on alleviating insulin tolerance were also weakened when co-treated with EX-527. c. Circulatory glucagon levels in the hucMSC and EX-527-treated group were higher than that in the hucMSC-treated group. d. Insulin levels in hucMSC- and EX-527-treated group were lower than the hucMSC-treated group. e. Immunofluorescence staining showed that when co-treated with EX-527, hucMSCs did not rescue islet structure. $f, g$. hucMSCs could not effectively restore $\alpha$ - and $\beta$-cell imbalance when co-treated with EX-527, as analyzed by Image-Pro Plus software ( $n=20-50$ islets per group). The results were expressed as the mean \pm SEM $(* P<0.05 ; * \star P<0.01 ; * \star * P<0.001)$.

\section{Supplementary Files}

This is a list of supplementary files associated with this preprint. Click to download.

- Additionalfile.docx 